\title{
Mass composition modelling at sources of ultra-high energy cosmic rays
}

\section{J. Olivares Schneider, ${ }^{a}$ J. Hoefken Zink ${ }^{a}$ and J. Bazo ${ }^{a, *}$}

${ }^{a}$ Sección Física, Departamento de Ciencias, Pontificia Universidad Católica del Perú Av. Universitaria 1801, Lima 32, Peru

E-mail: jbazo@pucp.edu.pe

Extragalactic sources are thought to be the origin of the highest energy cosmic rays detected at Earth. In this work, we explore energy-dependent mass compositions of these sources taking a subset of the VCV catalog for distances with $\mathrm{z} \leq 0.6$. We use a power-law spectrum with an exponential cutoff to model individual sources above $10^{18.7} \mathrm{eV}$. To be more realistic we use the individual distance and radio flux weight of the sources, while sharing other properties as the power law index, Z-dependent rigidity cut and nuclei fraction. We propagate the different nuclei using CRPropa and search for the best stable parameters that fit the features of the spectrum measured by the Pierre Auger Observatory. We show that the mass composition at the sources tends to have lighter nuclei, with $\mathrm{H}$ and He having the greatest contributions. The power law index found is $2 \pm 0.1$ and the rigidity cutoff $10^{21 \pm 0.3} \mathrm{eV}$.

*** The European Physical Society Conference on High Energy Physics (EPS-HEP2021), ***

*** 26-30 July $2021 * * *$

*** Online conference, jointly organized by Universität Hamburg and the research center DESY ***

${ }^{*}$ Speaker 


\section{Introduction}

Astrophysical cosmic ray sources are supposed to accelerate different nuclei up to extremely high energies. After propagation and interaction through the cosmic medium these nuclei and their products reach Earth. In this work we analyze the all-particle spectrum measured by the Pierre Auger Observatory (PAO) above the ankle [1]. There is evidence from PAO from the $X_{\max }$ distribution [2] that the mass composition of the ultra high energy cosmic rays (UHECR) is different from a pure proton composition.

Here we explore with simulations the mass composition at astrophysical sources taking a similar approach as done in [2]. Instead of a uniform source distribution, we apply real AGN source distances weighted by their radio flux. In the following, we describe how we model the sources, the catalogue selection, the propagation simulation, the method for obtaining the best model parameters to fit the PAO data and conclude with the results.

\section{Astrophysical sources modelling}

We consider that at the astrophysical sources a given proportion of different nuclei are accelerated. Assuming that the acceleration is electromagnetic we describe the injected spectrum as a nuclear $\left(Z_{A}\right)$ dependent power law with a broken exponential cut-off function [2]:

$$
\frac{d N_{A}}{d E}=f_{A} J_{0}\left(\frac{E}{10^{18} e V}\right)^{-\gamma} \times f_{\text {cut }}\left(E, Z_{A}, R_{\text {cut }}\right)
$$

where $f_{A}$ is the fraction of the injected nucleus over the total, $J_{0}$ a spectrum normalization, $\gamma$ the spectral index and $R_{\text {cut }}$ the maximum rigidity. The cut-off function, which limits the maximum acceleration energy, is defined by:

$$
f_{\text {cut }}\left(E, Z_{A}, R_{\text {cut }}\right)= \begin{cases}1 & \left(E<Z_{A} R_{\text {cut }}\right) \\ \exp \left(1-\frac{E}{Z_{A} R_{c u t}}\right) & \left(E>Z_{A} R_{\text {cut }}\right)\end{cases}
$$

In principle, each source could have different parameters. However, we are going to assume the same values for all sources to simplify the search.

\subsection{Sources selection}

Some possible cosmic ray sources have been identified assuming hadronic models using neutrino correlations in multi-messenger analyses [3-5]. These point towards blazars and radio-bright active galaxies.

As a more general approach, we start with the Véron-Cetty \& Véron catalogue of AGNs [6]. From there we select sources located with redshift $\mathrm{z} \leq 0.6$, since further away sources will not contribute in the final high energy spectrum at Earth. Then, the total number of selected sources is: $29.5 \mathrm{k}$. Since we are doing a 1D propagation analysis we group them by same redshift, giving 442 distances.

To get the final source distance distribution we weight each source by its radio flux ( $F_{20 \mathrm{~cm}} \mathrm{Jy}$ ). If the source has no radio flux, we take an average at the given distance. In Fig. 1 these distribution are shown. We see that by weighting with the flux the shape is changed towards larger distances. 


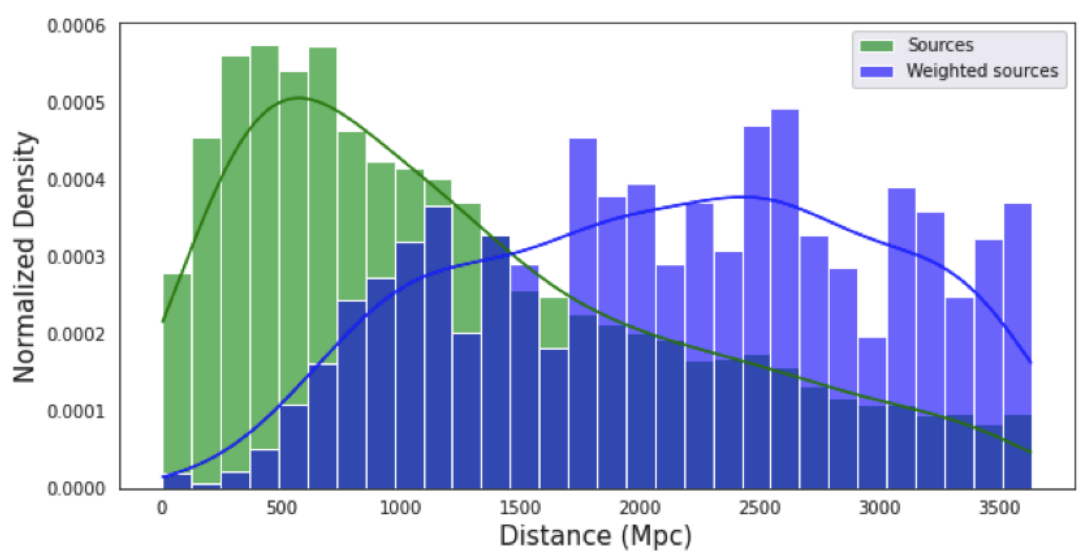

Figure 1: Astrophysical sources distance distribution in Mpc: unweighted (green) and weighted by their radio flux (blue).

\section{CRPropa Simulation}

We use the astrophysical simulation package CRPropa 3 [7] for propagating extraterrestrial ultra-high energy particles. We perform 1D simulations with minimum tracking energy of $1 \mathrm{EeV}$. The interactions set during propagation are photo-pion production, electron pair production and photo disintegration, all of them with CMB and IRB (Gilmore12 model) targets. We also include nuclear decay.

To simplify the simulations we inject only five representative stable nuclei in the same mass ranges used in [2]: hydrogen $\left({ }^{1} \mathrm{H}\right)$, helium $\left({ }^{4} \mathrm{He}\right)$, nitrogen $\left({ }^{14} \mathrm{~N}\right)$, silicon $\left({ }^{28} \mathrm{Si}\right)$ and iron $\left({ }^{56} \mathrm{Fe}\right)$. These starting nuclei can produce a larger set of nuclei after interactions during propagation.

For the event generation we proceed as follows. For values taken from the PDF of distances weighted with the radio flux (see Fig. 1) we randomly generate 10k energies from the broken exponential cut-off distribution (see Eq. 1) assuming identical sources and apply a nucleus fraction $\left(f_{A}\right)$ weight. Using this larger distance-energy distribution we simulate 750k events for each set of parameters during the best fit search.

\section{Best parameters search method}

Given the astrophysical source model we search for the best parameters that can fit the measurement of the all-particle energy spectrum of cosmic rays at Earth by PAO [1, 8]. We focus on data with energies above $10^{18.7} \mathrm{eV}$ (i.e. in the region above the ankle).

Since we are only going to compare shapes of the measured spectrum the parameters that we will fit are the spectral index $\gamma$, the rigidity cut-off $R_{c u t}$, and the mass fractions $f_{A}$ (i.e. four fractions, since the fifth is fixed by $\left.\Sigma f_{A}=1\right)$.

For the curve shape (normalized with $N_{\text {tot }}$ at Earth) distribution comparison between the number of $P A O$ and simulation $(\operatorname{sim})$ events $(N)$ at a given energy bin $(i)$ we apply the following 
$\chi^{2}$ which allows for few events in some bins:

$$
\chi^{2}=\sum_{i} \frac{\left(\frac{N_{i}^{P A O}}{N_{\text {tot }}^{P A O}}-\frac{N_{i}^{\text {sim }}}{N_{\text {tot }}^{\text {sim }}}\right)^{2}}{\frac{N_{i}^{P A O}}{N_{\text {tot }}^{P A O^{2}}}-\frac{N_{i}^{\text {sim }}}{N_{\text {tot }}^{\text {sim }}}}
$$

We look for the set of 6 parameters that give the minimum $\chi^{2}$, where $R_{c u t}$ and $\gamma$ are varied between $\left[10^{18}, 10^{24}\right] \mathrm{eV}$ and $[1,3]$, respectively.

\section{Results}

We perform a $6 \mathrm{D}$ grid search looking for the minimum $\chi^{2}$. When we analyze the obtained composition at the sources for $\chi^{2}<40$ (i.e. best 24 cases) we observe that heavier elements (i.e. $\mathrm{N}$, $\mathrm{Si}$ and $\mathrm{Fe}$ ) are highly suppressed. However, $\mathrm{H}$ and $\mathrm{He}$ can take a large number of values while still giving a good fit. The mean fractions of $\mathrm{H}$ and $\mathrm{He}$ are $0.65 \pm 0.31$ and $0.35 \pm 0.31$, respectively.

To refine the search we do a $2 \mathrm{D}$ grid search for $R_{c u t}$ and $\gamma$ with a fixed composition $\left(f_{H}=0.3\right.$, $f_{H}=0.7$ obtained for the best fit in the $6 \mathrm{D}$ grid search), shown in Fig. 2. We see a region of minima, with the global minimum at $R_{c u t}=10^{20.9} \mathrm{eV}$ and $\gamma=1.9$.

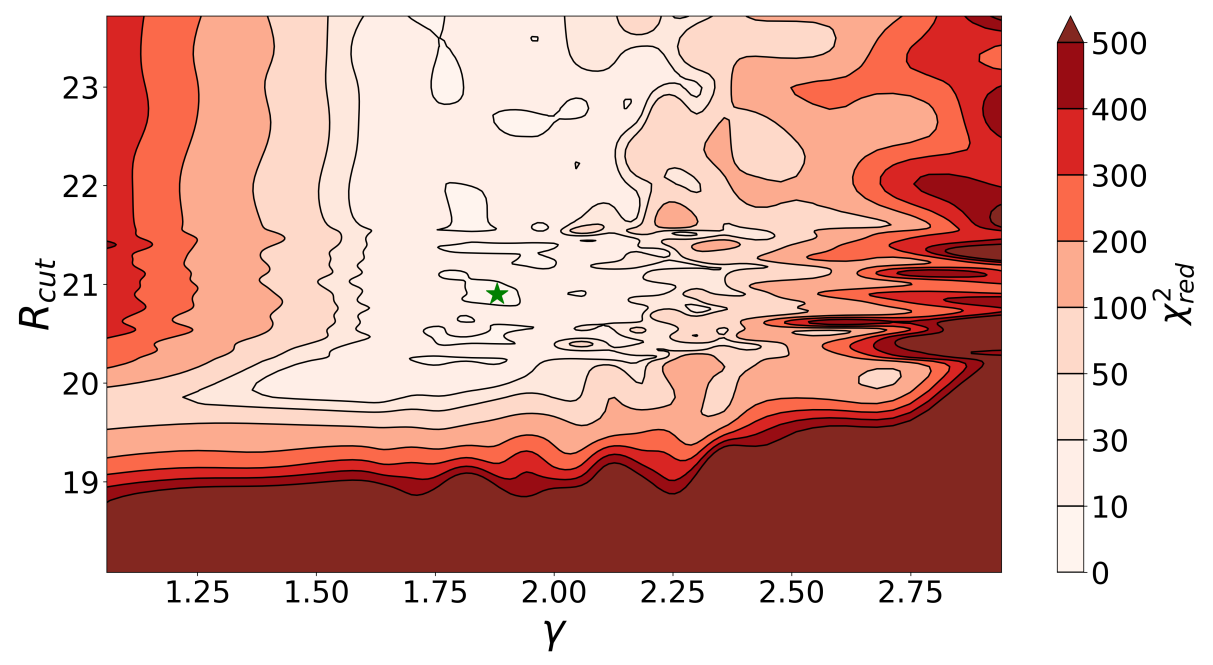

Figure 2: Best parameters search for $R_{\text {cut }}$ and $\gamma$ for a fixed set of nuclei fractions $\left(f_{H}=0.3, f_{H}=0.7\right.$, $\left.f_{N}=f_{S i}=f_{F e}=0\right)$. The color scale represents the reduced $\chi^{2}$. The global minimum is marked with a star.

Finally, we present in Fig. 3 the best fit $\left(\chi_{\text {red }}^{2}=2.87\right)$ of our model to the PAO all-particle data for energies greater than $10^{18.7} \mathrm{eV}$. There is a slight discrepancy at the highest energies. The composition found at Earth is also light being mostly protons with a smaller contribution from helium like nuclei.

\section{Conclusions}

We have used CRPropa 3 to simulate the propagation of high energy nuclei from astrophysical sources with precise 1D distance distributions of radio weighted AGNs. We model all sources with a unique power law spectrum with a broken exponential cutoff, which include 6 parameters to fit. 


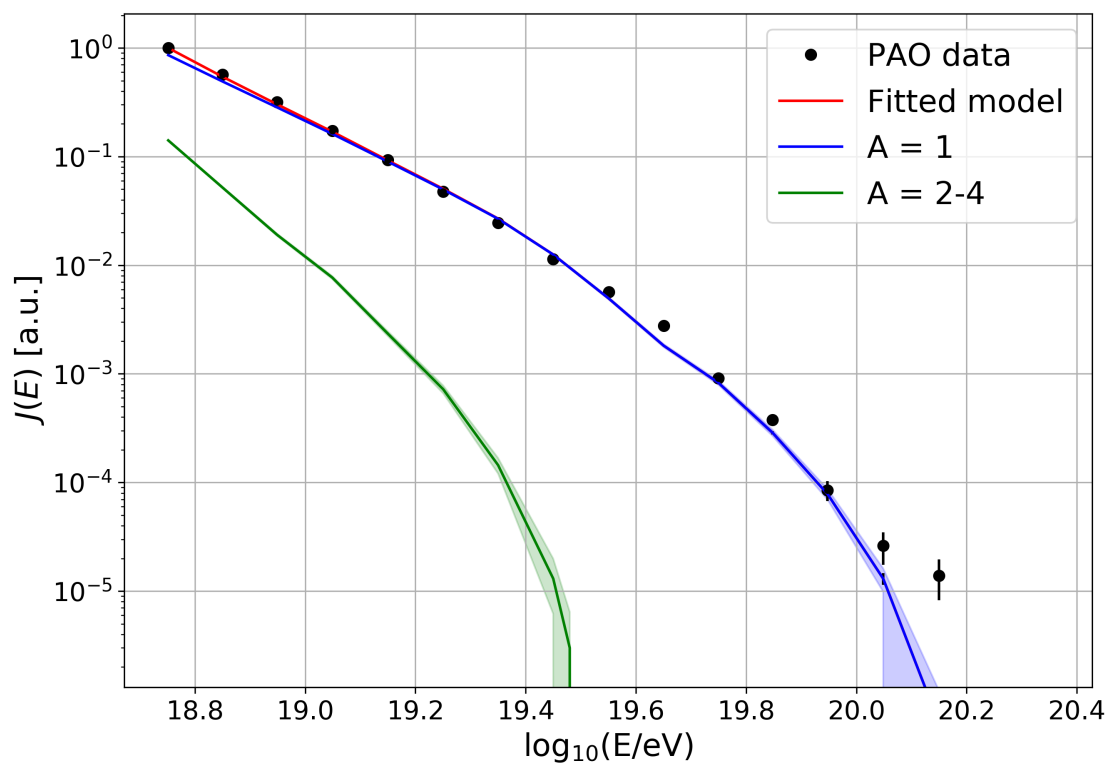

Figure 3: Comparison of PAO all-particle cosmic ray measure spectrum at Earth (black points) and best model (colored areas) $\left(R_{c u t}=10^{20.9} \mathrm{eV}\right.$ and $\gamma=1.9, f_{H}=0.3, f_{H}=0.7$, and $\left.\left.f_{N}=f_{S i}=f_{F e}=0\right)\right)$. The total model is shown in red, protons in blue and helium-like nuclei in green. The associated reduced $\chi^{2}$ is 2.87 .

The favoured mass composition at the sources that fits the PAO data $\left(E>10^{18.7} \mathrm{eV}\right)$ tends to have lighter nuclei: $\mathrm{H}$ and $\mathrm{He}$ have the greatest contributions with a possible small $\mathrm{N}$ composition. Heavier nuclei are almost rejected $(\mathrm{Si} \approx \mathrm{Fe} \approx 0)$. This is in agreement with the light composition $(\mathrm{H}+\mathrm{He})$ scenario found in [9]. However, in [2] they found a heavier chemical composition.

In addition, we find that the power law index is compatible with $2 \pm 0.1$ and the rigidity cutoff $10^{21 \pm 0.3} \mathrm{eV}$.

We are next planning to improve the analysis by including 3D simulations with galactic and extra-galactic magnetic fields and using different sets of possible cosmic ray sources (e.g. radio bright AGNs with neutrino correlations).

\section{Acknowledgements}

J. B. thanks the Dirección de Gestión de la Investigación (DGI - PUCP) for funding under Grant No. DGI-2019-3-0044.

\section{References}

[1] A. Aab and et al. (The Pierre Auger Collaboration). Measurement of the cosmic-ray energy spectrum above $2.5 \times 10^{18} \mathrm{eV}$ using the Pierre Auger Observatory. Physical Review D, 102(6), 2020. 
[2] A. Aab and et al. (The Pierre Auger Collaboration). Combined fit of spectrum and composition data as measured by the Pierre Auger Observatory. Journal of Cosmology and Astroparticle Physics, 2017(4), 2017.

[3] A. Plavin, Y.Y. Kovalev, Y.A. Kovalev, and S. Troitsky. Observational evidence for the origin of high-energy neutrinos in parsec-scale nuclei of radio-bright Active Galaxies. Astrophysical Journal, 894(2), 2020.

[4] A.V. Plavin, Y.Y. Kovalev, Y.A. Kovalev, and S.V. Troitsky. Directional association of TeV to PeV astrophysical neutrinos with radio blazars. Astrophysical Journal, 908(2), 2021.

[5] M.G. Aartsen and et al. Multimessenger observations of a flaring blazar coincident with high-energy neutrino IceCube-170922A. Science, 361(6398), 2018.

[6] M.-P. Véron-Cetty and P. Véron. A catalogue of quasars and active nuclei: 13th edition. Astronomy and Astrophysics, 518(22), 2010.

[7] R.A. Batista, A. Dundovic, M. Erdmann, K.-H. Kampert, D. Kuempel, G. Müller, G. Sigl, A.V. Vliet, D. Walz, and T. Winchen. CRPropa 3 - A public astrophysical simulation framework for propagating extraterrestrial ultra-high energy particles. Journal of Cosmology and Astroparticle Physics, 2016(5), 2016.

[8] A. Aab and et al. (The Pierre Auger Collaboration). Features of the energy spectrum of cosmic rays above $2.5 \times 10^{18} \mathrm{eV}$ using the Pierre Auger Observatory. Physical Review Letters, 125(12), 2020 .

[9] S. Das, S. Razzaque, and N. Gupta. Ultrahigh energy cosmic rays and neutrinos from light nuclei composition. Physical Review D, 99(8), 2019. 